\title{
Suffering Begets Suffering, and the Future of Primary Care
}

\author{
Stefan G. Kertesz, MD, MSc ${ }^{1,2}$ \\ 'Birmingham VA Medical Center, Birmingham, AL, USA; ${ }^{2}$ University of Alabama at Birmingham School of Medicine, Birmingham, AL, USA.
}

J Gen Intern Med 33(5):583-5

DOI: $10.1007 / \mathrm{s} 11606-018-4388-8$

(c) Society of General Internal Medicine (outside the USA) 2018

$\mathrm{T}$ wo papers in this issue delve into the interplay of illness, suffering, health care, and what policy advocates call the social determinants of health (Fig. 1). Both deliver wellcrafted evidentiary glimpses into how different forms of human suffering collide, overlap, and reinforce one another. Each tells a somewhat different tale, but leads to a similar quandary about whether our attributional models remain unequal to the task of understanding the suffering we health care providers and policymakers are called upon to address.

The first, by Charkhchi et al., looks at 24,480 American survey respondents with major medical conditions. All were respondents to the Behavioral Risk Factor Surveillance System (BRFSS) survey, with particular focus on respondents who had one of four illnesses (cancer, stroke, cardiovascular disease, and chronic lung disease). ${ }^{1}$ Secondarily, the authors tested the data for plausible effects of housing or food insecurity on health care access, and on health status itself. The latter purpose aligns closely with a proliferation of initiatives in which communities seek to address social determinants as an upstream intervention to avert health outcomes. $^{2}$

The BRFSS analyses revealed that housing insecurity (37\%) and food insecurity (31\%) were somewhat more common among persons who had major illnesses, compared to persons who did not (33\% for housing, and $24 \%$ for housing).

The adjusted odds of having food or housing insecurity were more pronounced for cardiovascular and lung disease, less pronounced for cancer and stroke. The authors suggest that stroke often proves so debilitating that survivors receive a surge of support, often moving in with family or into nursing facilities. In this way, persons suffering stroke obtain protection not consistently available for the other three illnesses.

In secondary analyses, housing and food insecurity were associated with double the odds of reporting difficulty accessing health care. Housing (but not food) insecurity was associated with lower odds of having good health status. The authors suggest that chronic illness affects both housing and food security, and that both may affect access to care and, possibly, health status.

Published online March 12, 2018
An alternative explanation, one among many, would suggest that some associations reflect causal vectors that sit outside the available data. For example, perhaps lung and cardiovascular disease are somewhat more robustly associated with housing and food insecurity as a result of behavioral risks, like tobacco. Such risks are more prevalent among Americans who are poor. From this point of view, one could speculate that a lack of economic power may, on average, predispose to (a) risk behaviors and (b) long-term economic hardship, both of which emerge in a statistical portrait of certain illnesses in the BRFSS. Many other hypotheses could be proposed.

The challenge of multiple compelling explanations applies with particular power to the present study of homeless adults by Patanwala et al. ${ }^{3}$ The authors had a different objective, which was to illustrate the burden of physical symptoms among a cohort of persons over the age of 50 who were currently or recently homeless ("homeless experienced" persons). The resulting paper reflects an extraordinary data collection process, in which the research team sought 350 currently homeless older adults living in the area around Oakland California, interviewed them extensively, and then followed them. It is rare to obtain so rich and rigorous a portrait of the increasingly old and sick people experiencing homelessness today.

Analytically this cohort reflects a daunting range of social, biological, environmental, and life history variables as plausible contributors to the experience of symptoms as basic as pain and difficulty sleeping.

Structuring their analysis is the Theory of Symptom Management, a framework developed by scholars of nursing. ${ }^{4}$

The results will not necessarily surprise readers but they are meaningful in more than one way. Descriptively, the HOPEHOME sample is vulnerable. Over half had experienced childhood abuse, and over half also experienced recent abuse. Symptom burden was high (one third qualified for moderatehigh physical symptom burden) and psychological symptoms, loneliness, and regret were extremely prevalent.

Multivariable analyses of these cross-sectional data suggested several variables were independently associated with symptom burden (female sex, childhood abuse, cannabis use, having $\geq 2$ chronic conditions, anxiety, hallucinations, and loneliness).

In reading both papers, a certain frustration is almost sure to arise. Some part of the reader seeks clear, linear associations between cardinal variables. This is, I suggest, our "inner epidemiologist." And even in reading the methods section 


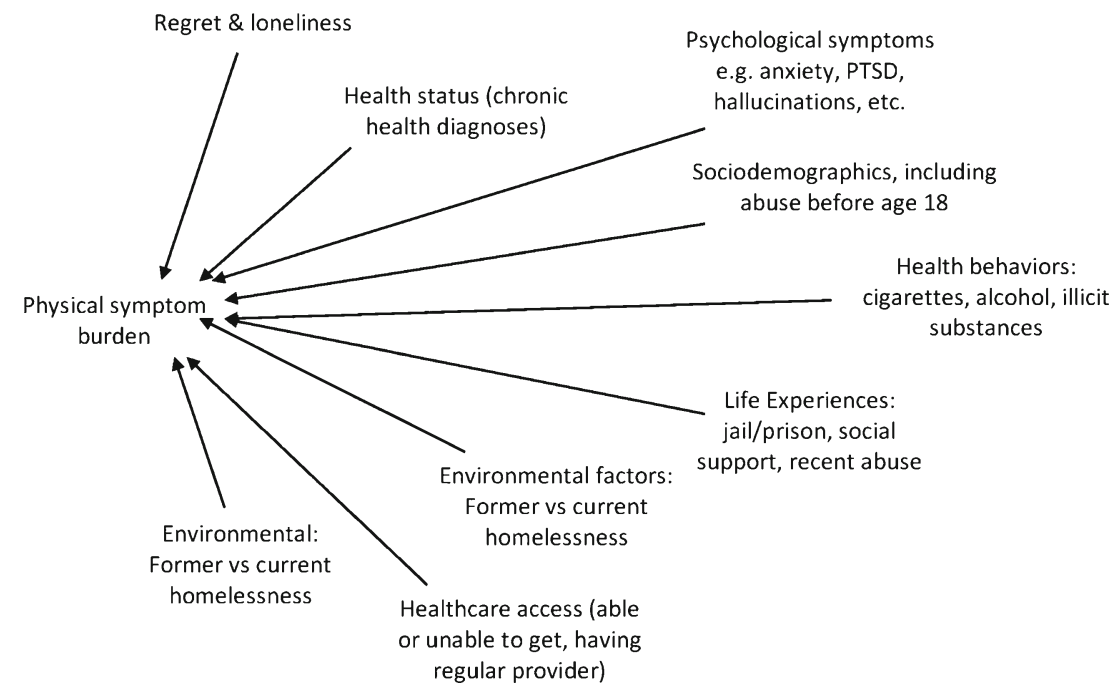

Fig. 1 Depiction of independent variables that may be associated with physical symptom burden among homeless adults assessed by Patanwala et al. ${ }^{3}$ optimized for graphic clarity and the convenience of the editorial writer. Arrows depicting potential bidirectional, direct, and indirect associations among these variables are not shown.

for both papers, my inner epidemiologist started to sweat. What should be seen as "cause," I asked myself, and what is really "effect." In preparing this comment, I drafted a graphic to help readers distinguish between dependent variable (symptoms, at left) and hypothesized independent predictors. The same exercise could have been done for the study by Charkhchi et al. My graphic plan was to put the dependent variable at far left, with "proximal" and "distal" causes splayed out from left to right, respectively. I was forced to give up as competing theories ricocheted and collided in my mind. For example:

Does substance use lead to job loss, which in turn exacerbates health conditions and symptoms? Or, do pain symptoms, intensified and rendered chronic by childhood abuse, somehow lead to job loss and homelessness, which in turn impede physical recovery? Or might homelessness induce substance use as a form of self-treatment, resulting in alienation from friends, loneliness, and an intensification of physical symptoms? No single interpretation seemed more credible than the other.

Ultimately, I decided that the only way to portray this web of suffering was to make sure the arrows from one variable did not graphically overlap the text referencing another. As a result, smaller text units like "regret" would necessarily be positioned closer on the page to the dependent variable, so that larger text blocks could get a clean shot at the same dependent variable, from further away on the page. So much for theory. Suffering begets suffering and this editorial is far from the first to notice. ${ }^{5}$

The frustration of the inner epidemiologist does not reflect a problem in either of these two papers. Rather, such frustration reflects an inevitable shortfall in how statistical models can account for the challenges afflicting populations we clinicians and policymakers wish to serve.

At this moment, a range of specific causes of human misery have captured some policymakers' attention as prime targets for fixing: lack of housing, loneliness, opioid prescriptions, and many more.

Each such cause deserves a rigorous scholarship, coupled to a practical policy response. But any response built on a single-target solution is likely to fall short or perhaps, prove counterproductive. A moral response to human suffering amidst unaffordable rental markets calls for expanded housing assistance, ${ }^{6}$ but that assistance cannot assure a return to health. ${ }^{7}$ Food insecurity hinders engagement in care, ${ }^{8}$ but so too does a failure to address unremitting symptoms, like pain. ${ }^{9}$ We who work in primary care are taught to think about whole people, but the imprecations of our managers and regulators undermine that, as they search for easy solutions to complex problems.

Our future in general internal medicine, most certainly in primary care, stands on the question of whether we will confront the complexity of each human being's suffering with full appreciation for the physical, social, emotional, and existential factors that differentiate one human situation from another and which, paradoxically, bind us together.

Corresponding Author: Stefan G. Kertesz, MD, MSc; University of Alabama at Birmingham School of Medicine, Birmingham, AL 35205, USA (e-mail: skertesz@uabmc.edu).

\section{Compliance with ethical standards:}

Conflict of Interest: The author declares that he has no conflict of interest.

\section{REFERENCES}

1. Charkhchi P, Dehkordy SF, Carlos RC. Housing and food insecurity, care access and health Status among the chronically ill: an analysis of the Behavioral Risk Factor Surveillance System. J Gen Intern Med. 2018. DOI:https://doi.org/10.1007/s11606-017-4255-Z 
2. Koo D, O'Carroll PW, Harris A, DeSalvo KB. An environmental scan of Recent initiatives incorporating social determinants in public health. Prev Chronic Dis. 2016; 13:E86.

3. Patanwala M, Tieu L, Ponath C, Guzman D, Ritchie CS, Kushel M. Physical, psychological, social, and existential symptoms in older homeless-experienced adults: an observational study of the HOPE HOME cohort. J Gen Intern Med. 2018. DOI: https://doi.org/10.1007/s11606017-4229-1.

4. Dodd M, Janson S, Facione N, Faucett J, Froelicher ES, Humphreys J, et al. Advancing the science of symptom management. J Adv Nurs. 2001;33(5):668-76.

5. Noor M, Shnabel N, Halabi S, Nadler A. When suffering begets suffering: the psychology of competitive victimhood between adversarial groups in violent conflicts. Pers Soc Psychol Rev. 2012;16(4):351-74.
6. Kushel M. Hepatitis a outbreak in California-addressing the root cause. N Engl J Med. 2018;378(3):211-3.

7. Kertesz SG, Baggett TP, O'Connell JJ, Buck DS, Kushel MB. Permanent supportive housing for homeless people-reframing the debate. N Engl J Med. 2016;375(22):2115-7.

8. Kushel MB, Gupta R, Gee L, Haas JS. Housing instability and food insecurity as barriers to health care among low-income Americans. J Gen Intern Med. 2006;21(1):71-7.

9. Upshur CC, Bacigalupe G, Luckmann R. "They don't want anything to do with you": patient views of primary care management of chronic pain. Pain Med. 2010;11(12):1791-8. 